\title{
AN ANALYTIC CHARACTERIZATION OF GROUPS WITH NO FINITE CONJUGACY CLASSES
}

\author{
E. R. COWIE
}

\begin{abstract}
Let $A$ be a unital Banach algebra and $\mathcal{G}$ the group of isometries in $A$. The norm in $A$ is uniquely maximal if $S$ is not contained in any larger bounded group in $A$ and there is no equivalent norm on $A$ with the same group of isometries. We use a group theory result of $\mathrm{B}$. $\mathrm{H}$. Neumann to prove that the discrete measure algebra $I^{\prime}(G)$ is uniquely maximal if and only if $G$ has no finite conjugacy classes.
\end{abstract}

1. Uniquely maximal norms. Let $A$ be a complex unital Banach algebra. Let $\mathcal{C}:=\left\{u \in A ;\|u\|=\left\|u^{-1}\right\|=1\right\}$. We shall call $\mathcal{G}$ the group of isometries in $A$. We shall say $A$ has a maximal norm if $\mathcal{G}$ is a maximal bounded group in $A$. This is equivalent to saying that there is no equivalent algebra norm on $A$ with more isometries. When $A$ is maximal, there may be many equivalent algebra norms on $A$ with the same group of isometries. What shall concern us in this paper is when a maximal norm is unique.

The unital Banach algebra $A$ has a uniquely maximal norm if it is maximal and there is no equivalent algebra norm on $A$ with the same group of isometries. The $B^{*}$-algebras are uniquely maximal. For commutative and finite dimensional algebras, uniquely maximal is equivalent to $B^{*}$ (see [1]). For a general unital Banach algebra we shall say the norm is convex transitive if

$$
\overline{\operatorname{co}}\{u ; u \in \mathcal{G}\}=\{a \in A ;\|a\| \leqslant 1\} .
$$

We have the following relationship between uniquely maximal and convex transitive:

TheORem 1. Let $A$ be a unital Banach algebra. Then $A$ is uniquely maximal if and only if $A$ is convex transitive and there does not exist $a \in A,\|a\|>1$ such that $\langle a,(\vec{i}\rangle$ (the semigroup generated by $a$ and $\mathcal{G}$ ) is bounded.

Proof. We shall first prove uniquely maximal implies convex transitive. We assume the norm is uniquely maximal. Suppose

$$
B=\overline{\operatorname{co}}\{u ; u \in \mathcal{G}\} \varsubsetneqq\{a \in A ;\|a\| \leqslant 1\} .
$$

Let $z \in A$ with $\|z\|=1$ and $z \notin B$. By the Hahn-Banach separation theorem (see [3, p. 60]), there exists $f \in A^{*}$, the dual space of $A$, such that $|f(u)| \leqslant 1$ for all $u \in \mathcal{Q}$

Received by the editors January 28, 1982

1980 Mathematics Subject Classification. Primary 20F38, 43A20, 46M05; Secondary 20F24.

Key words and phrases. Unital Banach algebras, maximal group, equivalent norms, uniquely maximal norm. convex transitive norm, finite conjugacy classes, centralizers, finite index, discrete measure algebras. 
and $f(z)>1$. Define

$$
\|x\|_{1}=\max \{\|x\|, \sup \{|f(u x)| ; u \in \mathcal{G}\}\} \text { for all } x \in A \text {. }
$$

Then $\|\cdot\|_{1}$ is an equivalent space norm on $A$. For $v \in \mathcal{G}$ we have

$$
\begin{aligned}
\|v x\|_{1} & =\max \{\|v x\|, \sup \{|f(u v x)| ; u \in \mathcal{G}\}\} \\
& =\max \{\|x\|, \sup \{|f(u x)| ; u \in \mathcal{G}\}\}=\|x\|_{1} .
\end{aligned}
$$

Let $\|\cdot\|_{2}$ be the operator norm from $\|\cdot\|_{1} ;\|a\|_{2}=\sup \left\{\|a x\|_{1} ;\|x\|_{1} \leqslant 1\right\}$. Then $\|\cdot\|_{2}$ is an equivalent algebra norm on $A$. For $v \in \mathcal{G}$ we have

$$
\|v\|_{2}=\sup \left\{\|v x\|_{1} ;\|x\|_{1} \leqslant 1\right\}=\sup \left\{\|x\|_{1} ;\|x\|_{1} \leqslant 1\right\}=1 \text {. }
$$

Hence, $\|\cdot\|_{2}$ has at least the same isometries as the original norm. As $A$ has a uniquely maximal norm, $\|a\|_{2}=\|a\|$ for all $a \in A$. In particular, we have

$$
\begin{aligned}
1 & =\|z\|=\sup \left\{\|z x\|_{1} ;\|x\|_{1} \leqslant 1\right\} \geqslant\|z\|_{1} \\
& =\max \{\|z\|, \sup \{|f(u z)| ; u \in \mathcal{G}\}\} \geqslant|f(z)|>1,
\end{aligned}
$$

a contradiction. Hence, $\overline{\operatorname{co}}\{u ; u \in \mathcal{G}\}=\{a \in A ;\|a\| \leqslant 1\}$, the norm is convex transitive.

Again assume $A$ is uniquely maximal. Suppose there exists $z \in A,\|z\|>1$ such that $\langle z, \mathcal{G}\rangle$ is a bounded semigroup. Define

$$
\|x\|_{1}=\sup \{\|b x\| ; b \in\langle z, \mathcal{G}\rangle\} \text { for all } x \in A .
$$

Then $\|\cdot\|_{1}$ is an equivalent space norm on $A$. Let $u \in\langle z, \mathcal{G}\rangle$, then

$$
\begin{aligned}
\|u x\|_{1} & =\sup \{\|b u x\| ; b \in\langle z, \mathcal{G}\rangle\}=\sup \{\|b x\| ; b \in\langle z, \mathcal{G}\rangle\} \\
& =\|x\|_{1} \text { for all } x \in A .
\end{aligned}
$$

As above we take the operator norm $\|a\|_{2}=\sup \left\{\|a x\|_{1} ;\|x\|_{1} \leqslant 1\right\}$ for all $a \in A$. This is an equivalent algebra norm on $A$ with at least the same isometries. As $A$ is uniquely maximal, $\|a\|_{2}=\|a\|$ for all $a \in A$. In particular, we have

$$
1<\|z\|=\sup \left\{\|z x\|_{1} ;\|x\|_{1} \leqslant 1\right\}=1 \text {, }
$$

a contradiction; hence, such a $z$ does not exist.

Let $\|\cdot\|_{1}$ be an equivalent algebra norm on $A$ with at least the same isometries. As $A$ is convex transitive, we have

$$
\{a ;\|a\| \leqslant 1\}=\overline{\operatorname{co}}\{u ; u \in \mathcal{G}\} \subseteq\left\{a ;\|a\|_{1} \leqslant 1\right\} .
$$

Hence, $\|a\|_{1} \leqslant\|a\|$ for all $a \in A$. Suppose there exists a $b \in A$ such that $\|b\|_{1}<$ $\|b\|$; by taking a suitable multiple we may assume $\|b\|_{1}<1<\|b\|$. But, we have $\langle b, \mathcal{G}\rangle \subseteq\left\{a ;\|a\|_{1} \leqslant 1\right\} \subseteq\{a ;\|a\| \leqslant K\}$ for some constant $K$ by equivalence. Hence, $\|b\|>1$ and $\langle b, \mathcal{S}\rangle$ is a bounded semigroup, a contradiction. Therefore $\|a\|_{1}=\|a\|$ for all $a \in A, A$ is uniquely maximal.

2. Groups with no finite conjugacy classes. Let $G$ be a group. Let the conjugacy class of $g \in G$ be denoted by $[g]=\left\{h^{-1} g h ; h \in G\right\}$ and the centralizer of $g$ by $C_{g}=\{h \in G ; h g=g h\}$. There is a one-to-one correspondence between the elements in $[g]$ and the cosets of $C_{g}$ in $G$. In particular, $[g]$ is finite $\Leftrightarrow C_{g}$ has finite index in $G$. 
Let $l^{1}(G)$ be the discrete measure algebra over $G$,

$$
l^{\prime}(G)=\left\{\sum \alpha(g) g ; \alpha(g) \in \mathbf{C}, \sum|\alpha(g)|<\infty\right\}
$$

with convolution multiplication, $\left(\sum \alpha(g) g\right)\left(\sum \beta(h) h\right)=\sum \gamma(k) k$ where $\gamma(k)=$ $\sum_{g h=k} \alpha(g) \beta(h)$ for each $k \in G$. The norm in $l^{\prime}(G)$ is given by $\left\|\sum \alpha(g) g\right\|=$ $\sum|\alpha(g)|$. The group of isometries in $l^{\prime}(G)$ is just the group, $\mathcal{G}=\{\lambda g ; g \in G$, $\lambda \in \mathbf{C}|\lambda|=1\}=G \times \mathrm{T}$. Obviously for any group $G, l^{\mathrm{l}}(G)$ has a convex transitive norm.

We shall prove that $G$ has no finite conjugacy classes if and only if $l^{\prime}(G)$ is uniquely maximal. In order to prove this result we shall require the following lemma, which may be of interest in itself. The lemma is based on the following interesting but little used group theory result of B. H. Neumann [2].

THEOREM 2. If a group $G$ is the set union of a finite number of cosets of subgroups, then one of those subgroups has finite index in $G$.

LEMMA 3. Let $G$ be a group with no finite conjugacy classes. If $S \subseteq G$ is a finite set with $n$ elements, then there exists $g \in G$ such that $S g S$ has precisely $n^{2}$ elements.

Proof. Let $S=\left\{g_{1}, \ldots, g_{n}\right\} \subseteq G$. Let $g \in G$, then $S g S=\left\{g_{i} g g_{k} ; 1 \leqslant i, k \leqslant n\right\}$. Fix $i, j, k$, and $l$, such that $i \neq k$ and $j \neq l$. Then

$$
g_{i} g g_{j}=g_{k} g g_{1} \Leftrightarrow g^{-1} g_{k}^{-1} g_{i} g=g_{1} g_{j}^{-1} \text {. }
$$

Let $H_{k i}=$ centralizer $\left\{g_{k}^{-1} g_{i}\right\}=\left\{h \in G ; h g_{k}^{-1} g_{i}=g_{k}^{-1} g_{i} h\right\}$ and $c_{i j k l} \in G$ be such that $c_{i j k l}^{-1} g_{k}^{-1} g_{i} c_{i j k l}=g_{l} g_{j}^{-1}$. The set of all $g$ which satisfy $(*)$ is the coset $H_{k i} c_{i j k l}$ or the empty set if no such $c$ exist. In order that $S g S$ has $n^{2}$ distinct elements, we require to choose $g$ not a member of all such $H c$ 's, i.e. show $G \neq \cup_{(i, j, k, l)} H_{k i} c_{i j k l}$. Suppose $G=\cup H c$, by Theorem 2, as this is a finite union of cosets, one of the subgroups has finite index in $G$. A centralizer $H$ of $G$ has finite index. Therefore either $G$ has a finite conjugacy class or $H=\operatorname{centralizer}\{e\}=G$. If $H=$ centralizer $\{e\}$ then $g_{1}=g_{k}$ for some $i, k$, but $i \neq k$ by definition. Hence, $G$ has a finite conjugacy class, a contradiction. Therefore $G \neq \cup_{(i, j, k, l)} H_{k i} c_{i j k l}$ and a suitable $g$ can be chosen.

COROLlaRY 4. Let $f \in l^{1}(G)$ be such thhat $f=\sum_{i=1}^{n} \lambda_{i} g_{i}$, then there exists $g \in G$ such that $\|f g f\|=\|f\|^{2}$.

We can now prove the main result of this paper.

THEOREM 5. The group $G$ has no finite conjugacy classes if and only if $l^{\prime}(G)$ has a uniquely maximal norm.

Proof. For the Banach algebra $l^{\prime}(G)$ the norm is always convex transitive. In order to prove $l^{\prime}(G)$ is uniquely maximal, we need only prove, by Theorem 1 , that there does not exist $a \in l^{\prime}(G)$ with $\|a\|>1$ such that $\langle a, \mathcal{G}\rangle$ is a bounded semigroup (where $\langle a, \mathcal{G}\rangle$ denotes the semigroup generated by $a$ and $\mathcal{G}$ the group of isometries in $\left.l^{\prime}(G)\right)$. Suppose such an $a$ exists. Let $K=\sup \{\|b\| ; b \in\langle a, \mathcal{G}\rangle\}$. Given $\varepsilon>0$, there exists $b \in\langle a, \mathcal{G}\rangle$ such that $1<K-\varepsilon<K-\varepsilon / 2<\|b\|<K$ for 
$\varepsilon$ sufficiently small. Now $b=\Sigma \lambda(g) g$ and $\|b\|=\Sigma|\lambda(g)|$. We can choose $\left\{g_{1}, \ldots, g_{n}\right\} \subseteq G$ such that $\left\|b-\sum_{i=1}^{n} \lambda\left(g_{i}\right) g_{i}\right\|<\varepsilon / 2$. Let $c=\sum_{i=1}^{n} \lambda\left(g_{i}\right) g_{i}$ and $d=b-c$. By Lemma 3 there exist $g \in G$ such that $c g c=\Sigma_{(i, j)} \lambda_{i} \lambda_{j} g_{i} g g_{j}$ has exactly $n^{2}$ distinct elements. Therefore

$$
\|c g c\|=\sum_{(i, j)}\left|\lambda_{i} \lambda_{j}\right|=\left(\sum_{i=1}^{n}\left|\lambda_{i}\right|\right)\left(\sum_{j=1}^{n}\left|\lambda_{j}\right|\right)=\|c\|^{2} .
$$

Now $\|c\| \geqslant\|b\|-\|b-c\|>K-\varepsilon / 2-\varepsilon / 2=K-\varepsilon>1$. Hence,

$$
\begin{aligned}
\|b g b\| & =\|c g c+c g d+d g c+d g d\| \geqslant\|c g c\|-\|c g d+d g c+d g d\| \\
& >(K-\varepsilon)^{2}-\varepsilon K+\varepsilon^{2} / 4=K^{2}-3 \varepsilon K+\frac{3}{4} \varepsilon^{2} .
\end{aligned}
$$

Hence, for $\varepsilon$ sufficiently small, $\|b g b\|>K$. This contradicts $K$ being the upper bound for the semigroup $\langle a, \mathcal{G}\rangle$. Hence, there does not exist $a \in l^{\prime}(G)$, with $\|a\|>1$ such that $\langle a, \mathcal{G}\rangle$ is a bounded semigroup, and the norm is uniquely maximal.

Conversely, suppose $g_{1} \in G \backslash\{e\}$ has a finite conjugacy class in $G ;\left[g_{1}\right]=$ $\left\{g_{1}, \ldots, g_{n}\right\}$. Let $a=g_{1}+\cdots+g_{n}$, then $g^{-1} a g=g_{1}+\cdots+g_{n}=a$ for all $g \in G$. Hence, $a \in \operatorname{centre}\left(l^{1}(G)\right)$. Let $b=a^{*}+i e+a=g^{-1}+\cdots+g_{n}^{-1}+i e+g_{1}$ $+\cdots+g_{n}$, then $b \in$ centre $l^{\prime}(G)$. Now $\|b\|=2 n+1$ but $\left\|b^{2}\right\| \leqslant(2 n+1)^{2}-2<$ $(2 n+1)^{2}=\|b\|^{2}$. Therefore $\rho(b)<\|b\|$, where $\rho$ denotes the spectral radius. By taking a suitable multiple of $b$ we may assume, $\rho(b)<1<\|b\|$. Hence, $b$ has bounded powers and as it is in the centre of $l^{\prime}(G),\langle b, \mathcal{G}\rangle$ is a bounded semigroup. Therefore, by Theorem $1, l^{\prime}(G)$ is not uniquely maximal.

\section{REFERENCES}

I. E. R. Cowie, Isometries in Banach algebras, Ph.D. thesis, Swansea, Wales, U.K., 1981.

2. B. H. Neumann, Groups covered by permutable subsets, J. London Math. Soc. 29 (1954), 236-248.

3. W. Rudin, Functional analysis, McGraw-Hill, New York, 1973.

Department of Mathematics, University College of Swansea, Singleton Park, Swansea Sa2 8PP, WALES, U.K.

Current address: Department of Physics, Mathematics and Computing, Manchester Polytechnic, All Saints, Manchester M15 6BH, U.K. 